\title{
Otrivine-Antistin-Pupil, Corneal and Conjunctival Responses to Topical Administration
}

\author{
D. R. TREW, L. A. WRIGHT, S. E. SMITH \\ London
}

\begin{abstract}
Summary
The ocular effects of Otrivine-Antistin eyedrops have been measured in a placebo controlled single-blind study in sixteen healthy volunteers. The drops produced mild sympathomimetic responses in the eye but had no effect on corneal sensitivity or on intraocular pressure. The evidence indicates that use of Otrivine-Antistin imposes no risk to the subject.
\end{abstract}

Otrivine-Antistin is a Pharmacy only eyedrop medication for the symptomatic relief of allergic or irritative conjunctivitis. It contains a sympathomimetic decongestant (xylometazoline hydrochloride 0.05 per cent $w / v)$ and an antihistamine (antazoline sulphate 0.5 per cent $\mathrm{w} / \mathrm{v}$ ) as active principles. Though it has also been in use for some years, the precise nature of the local responses to its application has been poorly documented.

A few studies have been conducted on the clinical use of sympathomimetic agents as ocular decongestants but they have concentrated on the subjective interpretation of effects on symptoms and signs. ${ }^{1,2}$ In addition, most have employed oxymetazoline as the sympathomimetic agent and its actions have been observed in animals. ${ }^{3}$ Objective tests, such as pupillometry, have occasionally been used but the results were inconclusive or not quoted. ${ }^{4,5}$

The use of combination drops in allergic conjunctivitis has been widespread and well accepted. ${ }^{6}$ Studies employing these preparations have shown their use in controlling symptoms and a good tolerance to administration by patients. ${ }^{7-9}$ However, Otrivine-Antistin eyedrops have not been included in the procedures. This study has been undertaken, therefore, to record the effects of these drops on pupil diameter, palpebral height, conjunctival vessel diameter, corneal sensitivity and intraocular pressure and to evaluate any possible risks associated with their use.

\section{Materials and Methods \\ Subjects}

Sixteen healthy subjects ( 8 male, 8 female) aged 19-59 years took part. All were of normal height and weight and none had significant abnormality on ocular, cardiovascular and general medical examination or in haematological or biochemical profiles. Each gave written consent to participate and the study was approved by the Ethics Committee of West Lambeth Health Authority.

\section{Treatments and Study Design}

Subjects attended on one occasion, in the morning. Otrivine-Antistin and a matching

Correspondence to: Mr D. R. Trew FRCS, Lecturer in Ophthalmology, Department of Clinical Pharmacology, St Thomas' Hospital, London SE1 7EH.

From: The Division of Pharmacological Sciences and Toxicology, United Medical and Dental Schools, St Thomas's Campus, London SE1 7EH. 
'vehicle only' solution were administered single blind as eyedrops. Two drops of drug solution were instilled 30 seconds apart to one eye and the same procedure followed for the drug-free vehicle to the second eye.

\section{Measurements}

Morning intraocular pressure values, for each subject, were recorded at a previous visit to the department by applanation tonometry, under benoxinate $0.4 \%$ local anaesthesia. This procedure was repeated at the conclusion of the experiment, i.e. 4 hours after eyedrop instillation. Adverse responses to the drops (stinging, irritation, misty vision, photophobia) were sought by direct questioning at 1, 5 and 10 minutes after instillation. At the same time, tearing and redness of the eyes were assessed by inspection.

The following measurements were performed before and at $0.5,1,1.5,2,3$ and 4 hours after eyedrop instillation:

(1) Pupil diameters were measured by infrared TV pupillography under bright illumination provided by two $30 \mathrm{~W}$ fluorescent lamps placed $50 \mathrm{~cm}$ from the subject. Six subjects were additionally evaluated under standard overhead lighting only.

(2) Eyelid positions were recorded with a

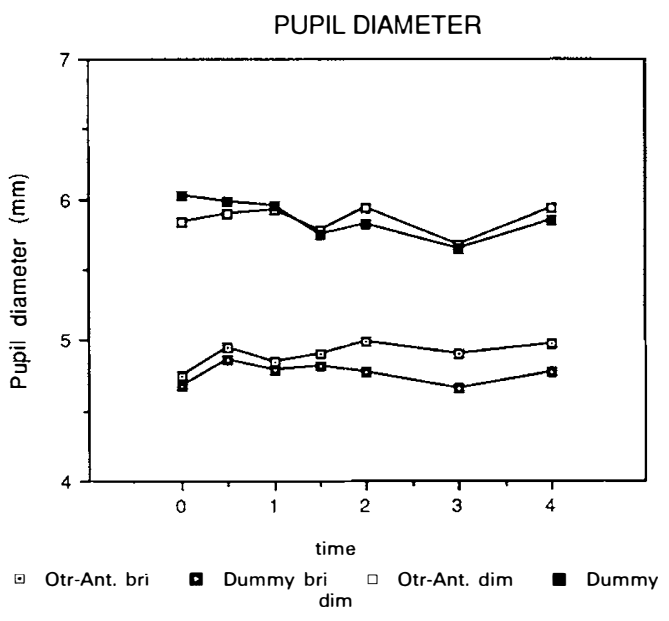

Fig. 1 Mean pupil diameters $(\mathrm{mm})$ for each trea tment at the two light levels. Open symbols represent OtrivineAntistin, closed symbols represent vehicle only. The upper graphs represent values in dim light $(n=6) ;$ the lower graphs represent values in bright light $(n=16)$.
Yashica Dental-Eye camera under flash illumination with the subject's gaze at infinity. The palpebral height was measured, on a projection, through the mid-point of the pupil.

(3) Conjunctival vessel diameter was measured from 1:1 photographs taken on the above camera. The negatives were viewed under a microscope using a calibrated eyepiece graticule. Corneal sensitivity was measured with a Cochet and Bonnet Aesthesiometer using a $0.12 \mathrm{~mm}$ filament.

\section{Statistical Analysis}

Values for pupil diameters, palpebral heights and conjunctival vessel diameters were analysed by comparisons between the two eyes of each subject using Student paired t-tests. Analysis of variance was also performed for all post-treatment values using these sources: subjects, eyes (treatments), times and interaction eyes-times. Intraocular pressure values were compared between eyes by paired t-tests and occurrences of symptoms and signs by Chi-squared tests.

\section{Results}

\section{Pupil Diameter}

Mean pupil diameters at the two lighting levels are illustrated in Figure 1. A small mydriatic response to Otrivine-Antistin occurred which was detectable only under bright illumination and which appeared to be maximal $(0.24 \mathrm{~mm})$ at 3 hours after instillation. Table I shows that the diameter difference was statistically significant but represented only an approximately 5\% change over the control eye.

\section{Palpebral Height}

Figure 2 shows the mean palpebral heights for each treatment. There was no inter-ocular difference before instillation, but OtrivineAntistin produced a small increase in palpebral height compared to the dummy treatment. The inter-ocular difference was detectable at 0.5 hour, reached a maximum at one hour $(0.57 \pm 0.14 \mathrm{~mm}, \quad \mathrm{P}<0.001)$ and declined thereafter. 
Table I Pupil diameter differences at each observation corrected for any initial differences, expressed as percent mydriatic response

\begin{tabular}{cccc}
\hline $\begin{array}{c}\text { Time (hr) } \\
\text { after } \\
\text { instillation }\end{array}$ & Mean \pm SEM & $\mathrm{t}$ & $\mathrm{P}$ \\
\hline 0.5 & $0.4 \pm 1.6$ & 0.227 & $\mathrm{NS}$ \\
1 & $0.5 \pm 1.5$ & 0.297 & $\mathrm{NS}$ \\
1.5 & $2.0 \pm 1.9$ & 1.055 & $\mathrm{NS}$ \\
2 & $3.9 \pm 2.0$ & 1.936 & $0.1>\mathrm{P}>0.05$ \\
3 & $4.6 \pm 2.1$ & 2.205 & $<0.05$ \\
4 & $3.0 \pm 1.6$ & 1.878 & $0.1>\mathrm{P}>0.05$ \\
\hline
\end{tabular}

Table II Mean values ( \pm SEM) for intraocular pressure ( $\mathrm{mm} \mathrm{Hg}$ ) obtained during screening and at 4 hours after eyedrop instillation

\begin{tabular}{lcc}
\hline Occasion & At screening & $\begin{array}{c}4 \mathrm{~h} \text { after } \\
\text { treatment }\end{array}$ \\
\hline Otrivine-Antistin & $15.4 \pm 0.3$ & $14.9 \pm 0.7$ \\
Dummy solution & $15.2 \pm 0.4$ & $14.9 \pm 0.6$ \\
Paired t & 0.824 & 0.124 \\
$\mathrm{P}$ & $\mathrm{NS}$ & $\mathrm{NS}$ \\
\hline
\end{tabular}

\section{Conjunctival Artery Diameter}

Figure 3 illustrates the mean diameters for single targeted arteries in each eye. Analysis of variance revealed that more than $50 \%$ of the variance was unidentifiable, which suggests that the data have poor repeatability, perhaps because the measurements were done at the limit of microscopic resolution. However, it can be seen that there is a trend towards vasoconstriction in response to Otrivine-Antistin, which was confirmed by analysis of variance. $(\mathrm{F}=6.005, \mathrm{P}<0.05)$.

\section{Intraocular Pressure}

Table II shows that Otrivine-Antistin produced no change in intraocular pressure by comparison with the dummy eyedrops.

\section{Corneal Sensitivity}

Median corneal sensitivity values (full filament extension) were unchanged by the treatments and in no subject was sensitivity impaired by either one.

\section{Symptoms and Signs}

Otrivine-Antistin produced some mild stinging, recorded at one minute after instillation, in $12 / 16$ and irritation in $5 / 16$ subjects; tearing and redness were observed in 4/16 and 6/16 subjects respectively. These effects had disappeared by 5 minutes. The dummy eyedrops produced temporary stinging, irritation and/or redness in only one subject. Only the difference in occurrence of stinging was statistically significant (Chi-squared $=12.955$, $\mathrm{P}<0.001)$.

\section{Discussion}

This study has shown that Otrivine-Antistin eyedrops produce very small, though statistically significant, changes in pupil diameter, palpebral height and conjunctival vessel diameter by comparison with dummy eyedrops containing only the vehicle. These

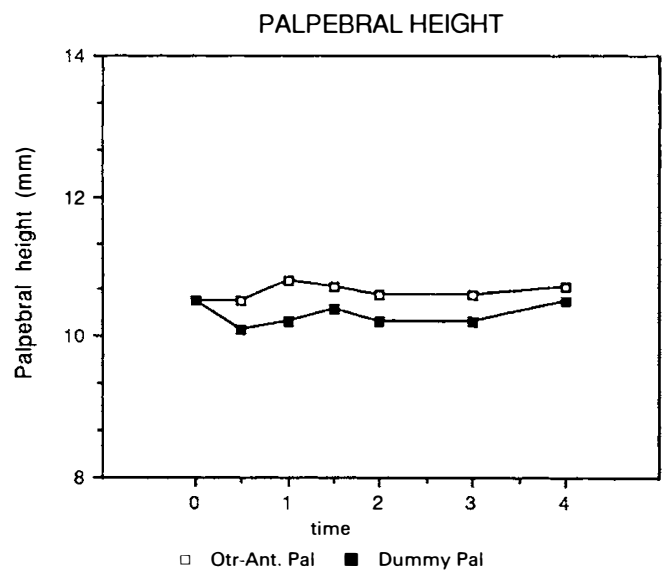

Fig. 2 Mean palpebral heights $(\mathrm{mm})$ for each treatment. Symbols as in Fig. 1.

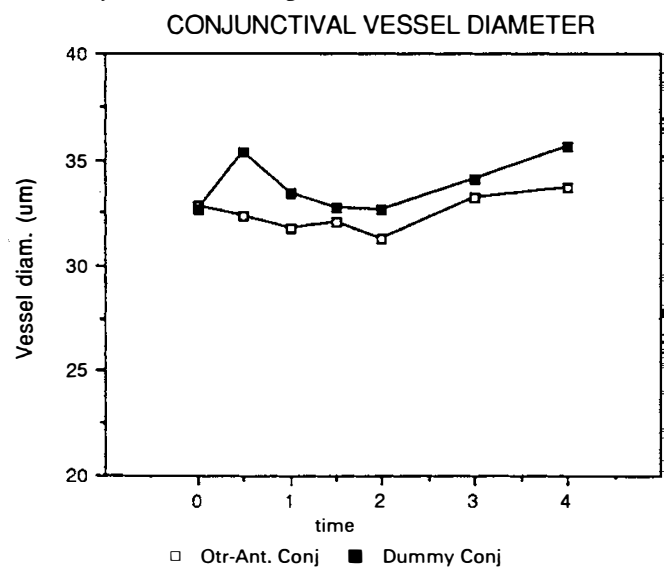

Fig. 3 Mean diameters (um) for single conjunctival arteries in each eye. Symbols as in Fig. 1. 
changes, comprising mydriasis, elevation of the upper eyelid and vasoconstriction, are consistent with a sympathomimetic response to the xylometazoline contained in the product. The mydriatic response was too small to be of clinical significance or to impose any risk of pupil block or irido-corneal angle closure and thus of acute glaucoma, even in susceptible subjects.

The eyedrops had no effect on corneal sensitivity or on intraocular pressure and produced some mild and transient irritation in some subjects, consistent with clinical experience.

We conclude that Otrivine-Antistin produces mild sympathomimetic responses in the eye but that in its use as a decongestant, these are harmless and impose no risk to the subject.

We thank Dr J. Gregory of Dispersa Ltd for the gift of Otrivine-Antistin eyedrops and for financial support. DRT is supported by the Iris Fund for Prevention of Blindness. DRT and LAW are supported by the Special Trustees of St Thomas' Hospital.

\section{References}

${ }^{1}$ Breakey AS, Cinotti AA, Hirshman M, Skowron RA, Samson CR, Danzig MR: A double-blind, multi-centre controlled trial of 0.025 per cent oxy- metazoline ophthalmic solution in patients with allergic and non-infectious conjunctivitis. Pharmatherapeutica 1980, 2: 353-6.

${ }^{2}$ Rybicza R and Mauracher E: Oxymetazoline ophthalmic solution versus naphazoline solution in non-infectious conjunctivitis. Pharmatherapeutica 1983, 3: 376-81.

${ }^{3}$ Duzman E, Anderson J, Vita JB, Lue JC, Chen CC, Leopold IH: Topically applied oxymetazoline. Ocular vasoconstrictive activity, pharmacokinetics and metabolism. Arch Ophthalmol 1983, 101: 1122-6.

${ }^{4}$ Fox SL, Samson CR, Danzig MR: Oxymetazoline in the treatment of allergic and non-infectious conjunctivitis. J Int Med Res 1979, 7: 528-30.

${ }^{5}$ Duzman E, Warman A, Warman R: Efficacy and safety of topical oxymetazoline in treating allergic and environmental conjunctivitis. $A n n$ Ophthalmol 1986, 18: 28-31.

${ }^{6}$ Abelson MB and Weston JH: Antihistamines. Ch 13 Clinical Ophthalmic Pharmacology. Ed. Lamberts DW, Potter DE. Boston and Toronto, Little, Brown and Co.

${ }^{7}$ Miller J and Wolf EH: Antazoline phosphate and naphazoline hydrochloride, singly and in combination for the treatment of allergic conjunctivitis-a controlled, double-blind clinical trial. Ann Allergy 1975, 35: 81-6.

${ }^{8}$ Abelson MB, Allansmith MR, Friedlaender $\mathrm{MH}$ : Effects of topically applied ocular decongestant and antihistamine. Am J Ophthalmol 1972, 90: 254-7.

${ }^{9}$ Lanier BQ, Tremblay N, Smith JP, de Faller JM: A double masked comparison of ocular decongestants as therapy for allergic conjunctivitis. Ann Allergy 1983, 50: 174-7. 